\title{
The enigmatic roles of microglial versus neuronal progranulin in neurological disease
}

\author{
Jason L. Eriksen
}

Received: 2 December 2009/Accepted: 3 December 2009/Published online: 12 December 2009

(C) Springer-Verlag 2009

Progranulin is an ancient, highly conserved gene that regulates cell division, survival, and migration across a wide variety of species. Until the publication of two papers showing that mutations in a single copy of the human $G R N$ gene was the cause of frontotemporal lobar dementia with ubiquitinated TDP-43 inclusions (FTLD-TDP) [2, 5] in 2006, very little was known about the importance of progranulin within the central nervous system. These reports created a strong impetus to identify new mutations and to explore the contribution of this protein in brain health and disease. Within 3 years of the first publications, the Alzheimer Disease and Frontotemporal Dementia Mutation Database (http://www. molgen.ua.ac.be/FTDMutations/) has grown to encompass 68 distinct $G R N$ mutations in 218 families.

In contrast to many neurodegenerative disorders where mutations result in a toxic gain-of function, most of the $G R N$-dependent FTLD-TDPs are thought to be caused by a global decrease in the levels of expressed or secreted progranulin. The vast majority of reported mutations lead to nonsense-mediated mRNA decay, a mechanism for elimination of mutant transcripts, resulting in complete loss of mRNA expression from one allele [7]. Although a variety of different mutations have been described, the most common genetic mutations result from premature insertion of stop codons or frame shifts, causing destruction of mRNA through nonsense-mediated decay. The prevailing wealth of mutations that have been recently discovered, all ascribed with a similar mode of action, have strongly supported the hypothesis that GRN haploinsufficiency results in insufficient GRN protein, leading to disease.

\section{J. L. Eriksen ( $\square)$}

Pharmacological and Pharmaceutical Science,

University of Houston, 521A SR2 4800 Calhoun,

Houston, TX 77204, USA

e-mail: jeriksen@central.uh.edu
Although a large number of mutations have been identified since 2006, there have been a paucity of studies characterizing GRN expression in FTLD-TDP patients, and the mechanisms describing the pathogenic loss of progranulin have been developed primarily from studies of patientderived lymphoblastoid cell lines. While these mechanisms clearly show the effect of the genetic mutations on production of the protein, there is a widespread variation in the age of disease onset; some carriers with pathological GRN mutations remain cognitively unimpaired into their 70s. Two publications in this issue of Acta Neuropathologica, one by Chen-Plotkin et al. [4] and the other by Naphade et al. [6], on the dynamic regulation of progranulin in the CNS suggest that the prevailing hypothesis of global GRN haploinsufficiency leading to a loss of protein may be too simplistic an explanation to completely account for development of FTLD-TDP.

The paper by Chen-Plotkin et al. [4] has filled a noticeable gap in the FTLD-TDP literature by providing a detailed comparison of regional progranulin expression in the brains of affected patients that span 11 different $G R N$ variants, comparing expression in frontal and temporal cortex, 2 histopathologically involved regions, and the occipital cortex and cerebellum, 2 spared regions, against the brains of both control patients and FTLD-TDP patients without GRN mutations. Similar to previous studies, the authors reported a notable reduction in $G R N$ transcripts in lymphoblastoid cell lines derived from the $G R N$ mutation carriers. However, when comparing levels of progranulin transcript between the normal controls and FTLD-TDP patients with GRN mutations, they made the surprising discovery that progranulin transcripts were not significantly decreased in any of the brain regions surveyed, and reported that the frontal cortex showed strongly elevated levels of GRN transcript compared with control. By 
sequencing brain mRNA from patients with GRN mutations, the authors showed that the remaining functional allele was responsible for increased expression. Semiquantitative immunoblot analysis of progranulin expression demonstrated that all four regions in FTLD-TDP brain showed a reduced expression of progranulin; but progranulin ELISAs of human brain lysates showed no significant differences between normal controls and GRN mutants in the highly involved frontal and temporal cortices, whereas there was a contrasting and significant reduction in progranulin in the occipital cortex and cerebellum. At first glance, these results seem contradictory to the hypothesis that $G R N$ haploinsufficiency is a cause of disease, but the authors used immunohistochemical studies to show that the majority of the increased GRN could be attributed to a large infiltration of microglia in the degenerating areas of the frontal and temporal cortices.

The other study by Naphade et al. [6], published in this issue, supports the similar conclusion that microglia contribute a substantial proportion of the total progranulin pool within the injured nervous system. In this paper, the authors used a well-characterized model of spinal contusion in C57BL/6 mice to study the time course of progranulin expression at both the messenger and protein levels, assessing expression over a 56 day time span. Results from the study showed that expression of progranulin at both the mRNA and protein levels peaked at 7 days and remained significantly elevated through 28 days. Detailed immunofluorescence studies demonstrated that increased progranulin expression strongly colocalized with $\mathrm{CD} 11 \mathrm{~b}+$ positive cells, a protein expressed on the surface of many leukocytes, including monocytes and macrophages. Using double immunofluorescence, the authors showed that progranulin colocalized with CD68, a lysosomal and endosomal marker strongly expressed in activated macrophages and microglia. Taken together, these data indicate that progranulin is strongly expressed by phagocytic macrophages and microglia in response to injury.

What conclusions can we draw from these two recent papers? Progranulin is expressed in both neuronal and microglial cells, and there are a number of papers that show progranulin transcripts become highly expressed during periods of neuronal injury and neurodegeneration. The data from these two papers suggest that the bulk of this GRN protein is contributed by the microglial population, and there appears to be little, if any, change in expression in the neuronal population with insult. What is perhaps most striking from these studies is the observation that while there is clearly a significant increase in progranulin contributed by the microglial population during injury, the increased level of progranulin protein does little to compensate for loss of progranulin expression in histopathologically involved areas, such as the frontal and temporal cortices.
In the periphery, the role of progranulin has often been cited as a secreted factor that is expressed under conditions of tissue remodeling where cells are dividing and actively migrating, during wound healing processes, and under inflammatory conditions [3], but the function of this protein, and whether it is secreted by neurons or microglia in the adult CNS is far less understood. One surprising observation that can be drawn from these recent papers is that there seem to be separately regulated pools of progranulin, expressed in neuronal and microglial populations, with non-overlapping biological activities. Such differences may explain why even highly overexpressed levels of progranulin in microglia are not sufficient to rescue neuronal degeneration caused by a loss of GRN transcripts in FTLD-TDP patients. Although the prevailing opinion in the field is that a global reduction in progranulin results in the development of FTLD-TDP, results from these papers suggest that progranulin levels are dynamically regulated in response to injury and neurodegeneration and can reach total levels that equal or exceed progranulin expression found in unaffected patients, mainly by increased expression of the remaining functional allele. In summary, these results suggest that the frontal and temporal cortices are specifically vulnerable to reductions in the available pool of neuronally expressed progranulin, and develop pathology independent of microglial progranulin expression.

The functional role of progranulin in neurodegeneration is only beginning to be explored, but the recent papers by Naphade et al. and Chen-Plotkin et al. provide thought provoking observations around the function of progranulin in the central nervous system. It is known that progranulin can serve as an anti-apoptotic factor in some non-neuronal cells [1], and if this relationship also holds true for the neuronal population, then the loss of the neuronal pool of progranulin may sensitize specifically vulnerable brain regions, such as the frontal and temporal cortices, to a broad range of environmental insults, resulting in widespread degeneration in instances of stress and trauma. Although work by Naphade et al. and Chen-Plotkin et al. have made some insightful observations into the regulation of progranulin in the nervous system, future studies will need to address what appears to be fundamental differences in the expression and the functional role of this protein in neurons and microglia.

\section{References}

1. Ahmed Z, Mackenzie IR, Hutton ML, Dickson DW (2007) Progranulin in frontotemporal lobar degeneration and neuroinflammation. J Neuroinflamm 4:7

2. Baker M, Mackenzie IR, Pickering-Brown SM, Gass J, Rademakers R, Lindholm C, Snowden J, Adamson J, Sadovnick AD, 
Rollinson S, Cannon A, Dwosh E, Neary D, Melquist S, Richardson A, Dickson D, Berger Z, Eriksen J, Robinson T, Zehr C, Dickey CA, Crook R, McGowan E, Mann D, Boeve B, Feldman $\mathrm{H}$, Hutton M (2006) Mutations in progranulin cause tau-negative frontotemporal dementia linked to chromosome 17. Nature 442:916-919

3. Bateman A, Bennett HP (2009) The granulin gene family: from cancer to dementia. Bioessays 31:1245-1254

4. Chen-Plotkin AS, Xiao J, Geser F, Martinez-Lage M, Grossman M, Unger T, Wood EM, Van Deerlin VM, Trojanowski JQ, Lee VM (2010) Brain progranulin expression in GRN-associated frontotemporal lobar degeneration. Acta Neuropathol. doi: 10.1007/s00401-009-0576-2
5. Cruts M, Gijselinck I, van der Zee J, Engelborghs S, Wils H, Pirici D, Rademakers R, Vandenberghe R, Dermaut B, Martin JJ, van Duijn C, Peeters K, Sciot R, Santens P, De Pooter T, Mattheijssens M, Van den Broeck M, Cuijt I, Vennekens K, De Deyn PP, Kumar-Singh S, Van Broeckhoven C (2006) Null mutations in progranulin cause ubiquitin-positive frontotemporal dementia linked to chromosome 17q21. Nature 442:920-924

6. Naphade SB, Kigerl KA, Jakeman LB, Kostyk SK, Popovich PG, Kuret J (2010) Progranulin expression is upregulated after spinal contusion in mice. Acta Neuropathol. doi:10.1007/s00401-009-0616-y

7. Rademakers R, Rovelet-Lecrux A (2009) Recent insights into the molecular genetics of dementia. Trends Neurosci 32:451-461 